\title{
Huntington's Disease with Retinitis Pigmentosa- a Case Report
}

\author{
R Mahmud ${ }^{1}$, M Habib ${ }^{2}$
}

\begin{abstract}
:
Huntington's disease (HD) is a chronic neurodegenerative disorder, characterized by the following triad of clinical hallmarks: autosomal dominant inheritance, choreoathetosis, and dementia. In 1993 the genetic mutation responsible for HD was identified and mapped on the chromosome 4p16.3. The mutation is a characteristic expansion of a CAG nucleotide triplet. In this paper we present a 36-years-old male patient with HD. Additionally he also had retinitis pigmentosa. His pedigree was reconstructed using available medical documentation and tracing other members of his family.
\end{abstract}

Key words: Huntington's Disease, Retinitis Pigmentosa.

\section{Introduction:}

Huntington's disease (HD) was described in 1872 by the American physician George Huntington, based on observations of patients attended by his father and grandfather in East Hampton, Long Island. The condition was designated "hereditary chorea."1

This disease is distinguished by the triad of autosomal dominant inheritance, choreoathetosis, and dementia. Its cause has been linked to abnormal expansion in a length of a CAG triplet repeat sequence in a gene on chromosome 4p, now called the Huntingtin gene (HD) ${ }^{2}$. Huntington's disease has a population frequency of about $7-10$ per 100,000 population and usually starting in adult life-fourth or fifth decade ${ }^{2}$. Huntington's disease manifesting in early adulthood is rare ${ }^{2}$. Juvenile onset HD is more severe form with repeat length of 60 or greater ${ }^{3}$.

As the disease progresses, chorea is gradually replaced by dystonia and parkinsonian features, such as bradykinesia, rigidity, and postural instability, which are usually more disabling than the choreic syndrome per se. In advanced disease, patients develop an akinetic-rigid syndrome, with minimal or no chorea.

1. Dr. Reaz Mahmud, MBBS, FCPS (Medicine), Junior Consultant (Medicine), Sarkari Karmachari Hospital, Dhaka.

2. Professor Mansur Habib, MBBS, FCPS (Medicine), MD (Neurology), MRCP (UK), FRCP (London), Head of the Department, Department of Neurology, Dhaka Medical College Hospital, Dhaka.

Address of correspondence :

Dr. Reaz Mahmud, MBBS, FCPS (Medicine), Junior Consultant (Medicine), Sarkari Karmachari Hospital, Dhaka.

Mob: +88-01912270803, E-mail: reazdmc22@yahoo.com
Other late features are spasticity, clonus, and extensor plantar responses. Dysarthria and dysphagia are common. Abnormal eye movements may be seen early in the disease. Other movement disorders, such as tics and myoclonus, may be seen in patients with $\mathrm{HD}^{4}$.

Retinitis pigmentosa (RP) is the most common form of hereditary retinal degeneration, affecting approximately 1 in 3500 individuals $^{5,6}$. It affects approximately 1.5 million people worldwide ${ }^{3}$. Classical RP is characterised by progressive night blindness and constriction of the peripheral visual fields, ultimately causing deterioration of the central vision in many patients.

Retinitis pigmentosa can be transmitted by all inheritance modes, with $\mathrm{X}$ linked recessive $\mathrm{RP}$ accounting for $10-20 \%$ of genetically identifiable cases and being reported among the most severe forms ${ }^{5.6}$.

In a patient presenting with Retinitis pigmentosa with choriform movement, common differential include Huntingon's disease-like 2, Neuroacanthocytosis, McLeod syndrome, Dentato Rubro Pallido LuysianAtrophy, (DRPLA), Hallervorden- spatz disease.

But even after extensive search we did not find any case report of huntngton's disease with retinitis pigmentosa. In this paper we present a 36-years-old male patient with HD. Additionally he also had retinitis pigmentosa. 


\section{Case report:}

A 36 year old male from Habiganj, sylhet presented in the Neurology department, Dhaka Medical Hospital with progressively increasing involuntary movement in the form of chorea involving all 4 limbs for 4 years. He also had history of progressive intellectual decline and forgetfulness for 3 years. He had occasional aggressive behavior. He also developed progressive loss vision in both eyes for last 2 years. Initially it was only impairment of vision at night but now his vision is limited to few meters.

He had a positive family history of such type of illness. His Father, one of his brothers and one nephew is affected. (Fig-1)

\section{Fig.1: Pedigree of the patient}

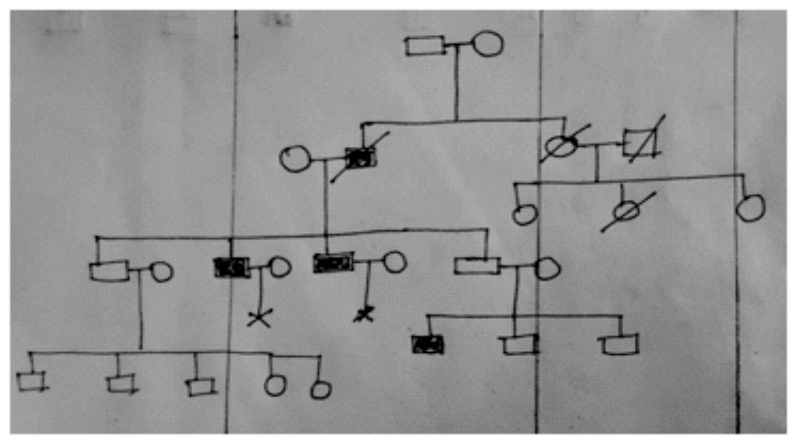

On examination patient was depressed, had choreiform movements all over the body. Neurologic examination revealed patient was conscious and oriented but MMSE score was 18. He was dysarthric. Cranial nerve examination revealed acuity of vision was limited to hand movement on both eyes with bilateral primary optic atrophy. There was retinitis pigmentosa on funduscopy. Motor examination revealed no weakness and in-coordination, planter was bilaterally flexor, there was no sensory deficit. Patient was unsteady during his walking.

\section{Fig:2 Fundal photograph of the patient}
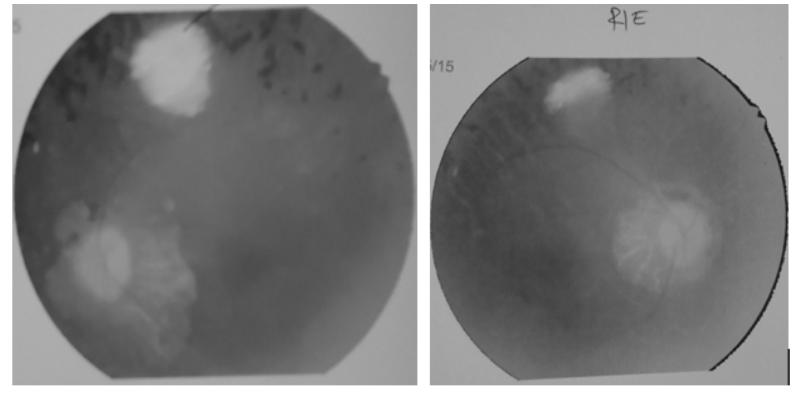

An impression of huntington's Disease was made and he was investigated to exclude possible differentials. His PBF revealed no acanthocytosis, wlison's screen was normal. MRI of Brain revealed atrophy of the caudate nuclei of brain. Genetic testing could not be done as it is not available in Bangladesh.

\section{Fig.3: MRI brain of the patient}

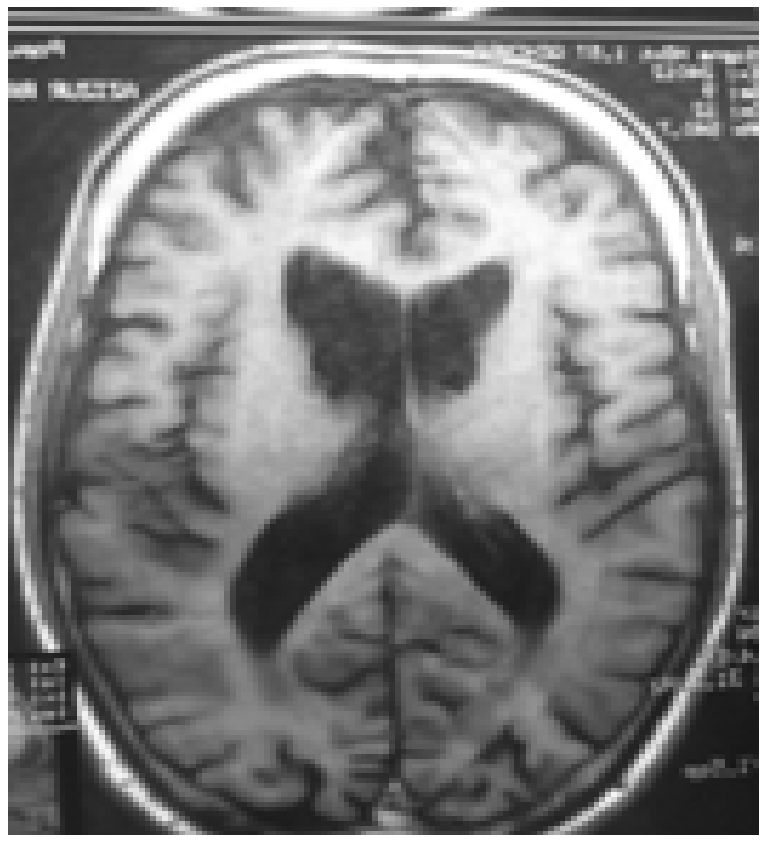

\section{Discussion:}

The diagnosis of huntington's dsease in this report was based on typical autosomal dominant pedigree, choreoathetosis, dementia and behavioral abnormality. His motor Diagnostic Confidence Level" (DCL) of the Unified Huntington's Disease Rating Scale (UHDRS) was 4 . So it may be labeled as Clinically Manifest HD (G10.2.3), not genetically confirmed. (Clinically manifest HD requires Motor DCL confidence 4 plus cognitive changes) ${ }^{7}$.

Our patient presented to some extent earlier than the typical age of presentation, this could be explained by anticipation as he remains second generation in the pedigree. (Fig-1)

The patient additionally had retinitis pigmentosa. (Fig2) So other genetic disease that could present with chorea and retinitis pigmentos like Neuroacanthocytosis, McLeod syndrome Dentato Rubro Pallido Luysian Atrophy (DRPLA) Hallervorden- spatz disease demands exclusion.

Neuroacanthocytosis is an autosomal recessive disease which is characterized by a beta lipoproteinemia, 
acanthocytosis, retinitis pigmentosa and pallidal degeneration. Our patient bears an autosomal dominant pedigree and his PBF revelaed no acanthocytoss. MRI of brain revealed caudate nucleus atrophy not pallidal atrophy.

McLeod syndrome is an $\mathrm{X}$ linked recessive disease and along with chorea, Dementia Acanthocyte in PBF it is commonly associated with axonal peripheral neuropathy. So it could be excluded by pedigree, absence of acanthocyte and peripheral neuropathy ${ }^{3}$.

Hallervorden- spatz disease is also an autosomal recessive disease and has mutation in PANK 2 gene. It commonly presents in childhood. The patient usually have pyramidal, extrapyramidal features in addition to dementia and retinitis pigmentosa. MRI could reveal a change in the signal intensity in the pallidum bilaterally $^{8}$ (eye of the tiger appearance) which in our patient was absent.

Dentato Rubral-PallidoLuysian Atrophy is autosomal dominant disease but it charactersticaly presents with cerebellar dysfunction along with chorea and dementia, which in our case is absent.

\section{Conclusion:}

So by the methods of exclusion this patient was labeled as clinically manifest Huntington's Disease. Genetic testing should be made available in our country, so that in case of such rare presentation we can confidently diagnose a patient.

\section{References :}

1. Huntington G. On Chorea. Med Surg Reporter. Philadelphia 1872; 26: $317-21$

2. Allan H, Martin A, Loushua P. Degenerative disease of the nervous system. In: Adams and victor's principle of Neurology. 9th ed. The McGraw Hill companies, 2009; p1027.

3. Robet BD, Gerald MF, Joseph J, John CM. Movement disorders. In: Bradley's Neurology in clinical practice. 6thed. Saunders: Elselvier Inc Philadelphia, 2012; p1786.

4. Ho A, Hocaoglu M. Impact of Huntington's across the entire disease spectrum: the phases and stages of disease from the patient perspective. Clin Genet. Sep 2011; 80(3):235-239.

5. Pagon RA. Retinitis pigmentosa. Surv Ophthalmol 1988; 33:137-77.

6. Berson EL. Retinitis pigmentosa: the Friedenwald lecture. Invest Ophthalmol Vis Sci. 1993; 34:1659-1676.

7. Ralf R, Blair R, Leavitt CM, Christopher AR. Diagnostic Criteria for Huntington's Disease Based on Natural History. Movement Disorders 2014; 29(11):1335-1341.

8. Allan H, Martin A, Loushua P, Allan H, Martin A, Loushua P. Inherited Metabolic disease of the Nervous System. In: Adams and victor's principle of Neurology. $9^{\text {th }}$ ed. The McGraw Hill companies, 2009; p943. 